\title{
Two-shape-tensor model for tumbling in nematic polymers and liquid crystals
}

\author{
Stefano S. Turzi \\ Dipartimento di Matematica, Politecnico di Milano, Piazza Leonardo da Vinci 32, 20133 Milano, Italy
}

(Received 14 March 2019; published 16 July 2019)

\begin{abstract}
Most, but not all, liquid crystals tend to align when subject to shear flow, while most nematic polymeric liquid crystals undergo a tumbling instability, where the director rotates with the flow. The reasons of this instability remain elusive, as it is possible to find similar molecules exhibiting opposite behaviors. We propose a continuum theory suitable for describing a wide range of material behaviors, ranging form nematic elastomers to nematic polymers and nematic liquid crystals, where the material parameters have meaningful physical interpretations and the conditions for tumbling emerge clearly. There are two possible ways to relax the internal stress in a nematic material. The first is the reorganization of the polymer network, the second is the alignment of the network natural axis with respect to the principal direction of the effective strain. We show that tumbling occurs whenever the second mechanism is less efficient than the first. Furthermore, we provide a justification of the experimental fact that at high temperatures, in an isotropic phase, only flow alignment is observed and no tumbling is possible, even in polymers.
\end{abstract}

DOI: 10.1103/PhysRevE.100.012706

\section{INTRODUCTION}

Nematic liquid crystals and nematic polymers either undergo shear aligning or tumbling when subjected to a simple shear flow. Shear aligning is characterized by a director dynamics that evolves monotonically to a fix orientation at steady state. By contrast, tumbling occurs when the director undergoes periodic oscillations in its orientation with a period that is inversely proportional to the shear rate. The classical Ericksen-Leslie model of nematic liquid crystals (NLCs) predicts that the tendency of a liquid crystal to either tumble or flow align is controlled by the sign of the ratio of two viscosity coefficients: $\alpha_{3} / \alpha_{2}$. Positive values lead to flow aligning and negative values cause tumbling.

However, the different sign of $\alpha_{2}$ and $\alpha_{3}$ has no clear chemical or physical interpretation. Thus, a number of possible explanations for such a dramatic difference in the flow dynamics has been proposed in the literature over the years. It had long been thought that prolate nematogens always align with the flow, then it was discovered that some nematic liquid crystals undergo a tumbling instability in part of their nematic range. By contrast, most side-chain polymer liquid crystals show tumbling most of the times [1].

Furthermore, for some compounds, these different behaviors are unexpected since their molecular structure and their phase diagrams are very similar [2-4]. For example, while MBBA and 5CB always flow align in their nematic phase, other closely related molecules, respectively $\mathrm{HBAB}$ and $8 \mathrm{CB}$, undergo a transition from flow alignment to tumbling when the temperature is decreased below a given threshold [2,3].

This instability has been associated with smectic fluctuations in the nematic phase [5], with strong side-to-side molecular aggregation [6,7] and, in other theories [8], the rotational friction, the order parameter strength, and molecular form factors play a key role. Many theories of nematic liquid crystals fail to predict the transition from flow aligning to tumbling behavior. Some retain the transition, but the interpretation they provide for the tumbling parameter is not widely accepted.

One successful strategy to relate the viscous response of NLCs to the effective mesoscopic features of the microscopic constituents, is to derive a kinetic theory of NLCs. This was originally developed by Kuzuu and Doi [9-11] and then extended by Osipov and Terentjev [8,12,13] and Larson [2,7,14]. In general, kinetic theories require specific assumptions on the intermolecular potential, and this choice particularly affects the antisymmetric part of the Cauchy stress tensor, which is responsible for the director rotations. Furthermore, these theories usually include higher order moments of the orientational distribution function and some closure approximations are usually necessary to make the theory more tractable. However, the most obvious closure approximations imply that NLCs always exhibit flow aligning [14], thus more sophisticated closure approximations are often used to be able to include the tumbling effect. Finally, kinetic theories neglect the analysis of the translational molecular degrees of freedom, which give a fundamental contribution to the Newtonian viscosity (the Leslie coefficient $\alpha_{4}$ ).

In this paper, we propose an alternative route to include far from equilibrium effects, such as tumbling, into a continuum theory. Namely, we develop a "mixed" theory where the macroscopic degrees of freedom are treated classically, but the microscopic degrees of freedom are taken into account in a coarse grained way by introducing material reorganization and relaxation. The advantage of this approach is its simplicity, the guiding principles being material symmetry and irreversible thermodynamics. While in this case not all the microscopic details can be accounted for (like in kinetic theories, however), nonetheless, we get a better insight into the microscopic mechanisms underlying tumbling phenomena.

It is worth remarking that possibly many microscopic phenomena, such as molecular interactions or molecule flexibility, may affect the value of our material parameters. As 
such, these parameters are not directly related to specific microscopic mechanisms, but have to be considered as effective model parameters, whose value is the result of a number of complicated microscopic interactions that we do not wish to model in detail. Hence, the validity of our model is essentially limited to those situations where the effective material parameters can be considered independent of the imposed macroscopic flow.

The paper is organized as follows: The theory is described in Secs. II and III. In Secs. IV and V we obtain some consequences of the theory, in the approximation of fast relaxation times. Specifically, we derive how the Leslie coefficients depend on the model parameters and discuss the tumbling phenomenon. The conclusions are drawn in Sec. VI. Finally, some mathematical details on the derivation are reported in the Appendices.

\section{NATURAL POLYMER NETWORK}

In our previous papers [15-17] we have shown how nematic elastomers (NEs), nematic liquid crystals (NLCs), and nematic polymers (NPs) can be described, at the continuum level, by the same theory. A posteriori, this is not surprising since they share the basic features of a continuum theory, namely, material symmetry and compatibility with thermomechanics principles.

If we model nematic elastomers as rubbery networks with an aligned uniaxial anisotropy of their polymer strands and with a coupling to the nematic mesogenic units, the transition from a elastic response of NEs to a fluidlike behavior of NLCs is obtained by allowing the polymer network to reorganize. Hence, we consider a transient polymer network, where cross links can break under stress at some rate and reform in an unstressed state, so that the network undergoes a plastic deformation to reach a natural state with zero stress, a state that we call natural (or relaxed) polymer network. However, at short timescales, when the cross links are not broken, the material is elastic. In general, when the cross-linking rate is much higher than the breakage rate, the network can be regarded as "cross linked," or elastic. When the two rates are comparable, the system undergoes a plastic flow under stress and when the cross-linking rate is much lower than the breakage rate, the system quickly relaxes to a natural state and it behaves like a viscous fluid. Of course, in real nematic liquid crystals the network is not physical, but it is only an idealization. Its transient nature mimics the rearrangement of the position of the nematic molecules that typically takes place in fluids.

Usually, the positional order of the cross links or of the nematic molecules, at each instant of time, is only known via some averaged quantities. A standard simplification in this respect is that the second moment tensor is sufficient to describe the positional distribution of the molecules. Hence, in analogy with nematic elastomer theory [18], we define a shape tensor $\boldsymbol{\Psi}_{*}$ as the chain step-length tensor of the relaxed network (or, depending on interpretation as the normalized covariance tensor of the one-particle probability density of the position of the molecules)

$$
\boldsymbol{\Psi}_{*}\left(\rho, \mathbf{n}_{*}\right)=a(\rho)^{2}\left(\mathbf{n}_{*} \otimes \mathbf{n}_{*}\right)+a(\rho)^{-1}\left(\mathbf{I}-\mathbf{n}_{*} \otimes \mathbf{n}_{*}\right),
$$

where $\rho$ is the density, $a(\rho)$ is a shape parameter that gives the amount of spontaneous elongation along the main axis $\mathbf{n}_{*}$. When $a(\rho)=1$, the centers of mass distribution is isotropic, while for $a(\rho)>1(<1)$ it is prolate (respectively, oblate) in the direction of $\mathbf{n}_{*}$. The normalization condition corresponds to the requirement $\operatorname{det}\left(\boldsymbol{\Psi}_{*}\right)=1$ since we are only concerned with the anisotropy of the molecular distribution. Our definition of $\boldsymbol{\Psi}_{*}$ mimics the definition of step-length tensor that is used in nematic elastomer theory to describe the anisotropic polymer ordering and represents the spontaneous stretch of the material.

The interpretation of the shape tensor for LCs is more indirect. Since LCs can be seen as a particular case of relaxing nematic elastomers, when the relaxation time is much shorter than all the other times involved [15,16,19], the physical interpretation of $\boldsymbol{\Psi}_{*}$ for LCs is deduced as a limiting case from that of the nematic elastomers. A more direct interpretation requires the introduction of the one-particle probability density of the position of the molecules in nematic liquid crystals. As shown in molecular simulations, this is slightly anisotropic, i.e., on average, the molecules are more distant along the direction of the director and are more densely packed in a direction orthogonal to the director. This defines a new direction of anisotropy that is usually strongly correlated with the nematic director, but conceptually distinct.

In standard nematic elastomer theory, and in our previous works $[15,16]$, it is assumed that the relaxed network main axis $\mathbf{n}_{*}$ is directed along the nematic director $\mathbf{n}$, at each instant of time. In so doing, the director is taken to describe, at the same time, the preferred orientation of the molecules and the relative distance of their centers of mass at equilibrium (or the direction of the natural strain in the network). This assumption is valid for most NLCs and for NEs and leads to interesting consequences such as the connection of the Leslie coefficients with the elastic features and the relaxation times of the material, the dependence of viscosity coefficients on frequency, and the viscoelastic response of the material. Furthermore, a new Parodi-type relation is identified for NLCs which seems to be in good agreement with molecular simulations and in fairly good agreement with experiments.

However, the assumption of an instantaneous relaxation of $\mathbf{n}_{*}$ to the director $\mathbf{n}$ leads to a flow-aligning director field and prevents tumbling instability, which is observed in most nematic polymers and in some liquid crystals. Therefore, a key ingredient for the presence of tumbling seems to be the distinction between $\boldsymbol{\Psi}_{*}$ and the elastonematic-coupling tensor defined as

$$
\boldsymbol{\Psi}(\rho, \mathbf{n})=a(\rho)^{2}(\mathbf{n} \otimes \mathbf{n})+a(\rho)^{-1}(\mathbf{I}-\mathbf{n} \otimes \mathbf{n}),
$$

the only difference with respect to (1) being the substitution of $\mathbf{n}_{*}$ with $\mathbf{n}$ (see Fig. 1). Our description differs at this point from the standard elasticity of nematic elastomers where there is a direct coupling between the director field and the polymer network. Instead of a single shape tensor, here we introduce two closely related tensors $\boldsymbol{\Psi}_{*}$ and $\boldsymbol{\Psi}$, and the coupling between their axes $\mathbf{n}_{*}$ and $\mathbf{n}$ is described by an energetic term that favors the alignment of $\mathbf{n}_{*}$ with $\mathbf{n}$. As we shall see, this introduces an additional governing equation and a corresponding characteristic time. 


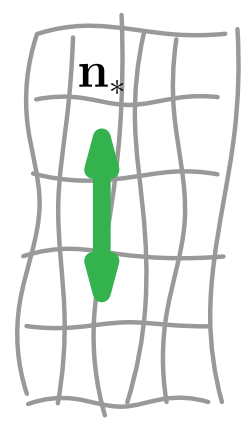

$\Psi_{*}$

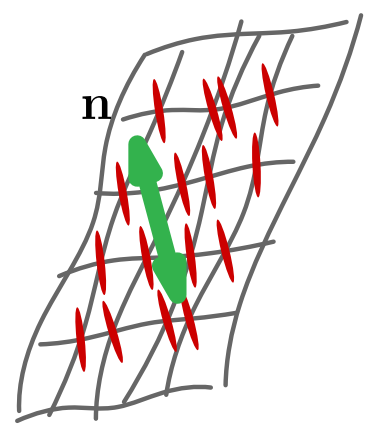

$\mathrm{B}_{\mathrm{e}}$
FIG. 1. Schematic representation of the natural network, with main axis $\mathbf{n}_{*}$, whose anisotropy is described via the shape tensor $\boldsymbol{\Psi}_{*}$ (left). For comparison, on the right-hand side we show the schematic representation of the deformed actual transient network, with effective strain tensor $\mathbf{B}_{\mathrm{e}}$. The material is stress free when $\mathbf{B}_{\mathrm{e}}$ coincides with $\boldsymbol{\Psi}_{*}$. The director $\mathbf{n}$ describes the average direction of the molecules and is an additional degree of freedom, but it is energetically coupled with the axis $\mathbf{n}_{*}$.

To account for the transient nature of the network (or material reorganization, in the case of NLCs), we split the deformation gradient $\mathbf{F}$ into elastic part $\left(\mathbf{F}_{\mathrm{e}}\right)$ and relaxing part (G), and define

$$
\begin{gathered}
\mathbf{F}_{\mathrm{e}}=\mathbf{F G}^{-1}, \quad \mathbf{H}=\left(\mathbf{G}^{T} \mathbf{G}\right)^{-1}, \\
\mathbf{B}_{\mathrm{e}}=\mathbf{F}_{\mathrm{e}} \mathbf{F}_{\mathrm{e}}^{T}=\mathbf{F G}^{-1} \mathbf{G}^{-T} \mathbf{F}^{T}=\mathbf{F} \mathbf{H} \mathbf{F}^{T},
\end{gathered}
$$

where $\mathbf{B}_{\mathrm{e}}$ is the effective left Cauchy-Green deformation tensor, and $\mathbf{H}$ is the inverse relaxing strain tensor.

Furthermore, we posit the following free-energy density per unit mass

$$
\begin{aligned}
\sigma( & \left., \mathbf{B}_{\mathrm{e}}, \mathbf{n}_{*}, \mathbf{n}, \nabla \mathbf{n}\right) \\
= & \sigma_{0}(\rho)+\frac{1}{2} \mu_{1}(\rho)\left[\operatorname{tr}\left(\boldsymbol{\Psi}_{*}^{-1} \mathbf{B}_{\mathrm{e}}-\mathbf{I}\right)-\log \operatorname{det}\left(\Psi_{*}^{-1} \mathbf{B}_{\mathrm{e}}\right)\right] \\
& +\frac{1}{2} \mu_{2}(\rho) \operatorname{tr}\left(\boldsymbol{\Psi}_{*}^{-1} \boldsymbol{\Psi}-\mathbf{I}\right)+\sigma_{\mathrm{Fr}}(\mathbf{n}, \boldsymbol{\nabla} \mathbf{n}) .
\end{aligned}
$$

The first term $\sigma_{0}(\rho)$ penalizes volume changes; it is assumed to be large and not depending on material relaxation. The second term represents a neo-Hookean energy where the natural (zero stress) deformation is described by $\boldsymbol{\Psi}_{*}$ and is, therefore, transversely isotropic in the direction of $\mathbf{n}_{*}$. This free-energy term only depends on the effective tensor $\mathbf{B}_{\mathrm{e}}$ which is allowed to relax to its natural state $\boldsymbol{\Psi}_{*}$. The bulk modulus associated with the elastic response is $\rho \mu_{1}(\rho)$. However $\mathbf{n}_{*}$ (and hence $\boldsymbol{\Psi}_{*}$ ) is not fixed, but can rotate in order to align with the director $\mathbf{n}$. This contribution is encoded in the third term which penalizes any deviation of $\mathbf{n}_{*}$ from the director $\mathbf{n}$. The relative importance of this term with respect to the second one is determined by the ratio of their elastic moduli: $\mu_{2} / \mu_{1}$. Finally, we consider a Frank elastic-potential $\sigma_{\mathrm{Fr}}(\mathbf{n}, \nabla \mathbf{n})$ that favors the alignment of nematic molecules and whose prototype is $\sigma_{\mathrm{Fr}}(\mathbf{n}, \nabla \mathbf{n})=k|\nabla \mathbf{n}|^{2}$. For more complex scenarios in this respect, the reader is advised to consult Ref. [20].
Intuitively, the dynamics of $\mathbf{n}_{*}$ is governed by two independent contributions: on the one hand, $\mathbf{n}_{*}$ is coupled to the effective macroscopic deformation $\mathbf{B}_{\mathrm{e}}$, so that $\mathbf{n}_{*}$ tends to align with a principal direction of the effective strain; on the other hand, $\mathbf{n}_{*}$ tends to coincide with the director $\mathbf{n}$. The director $\mathbf{n}$ is an additional degree of freedom, so if there was no Frank potential, it would always be favorable to make the director align with the major axis of $\mathbf{B}_{\mathrm{e}}$, and take $\mathbf{n}_{*}=\mathbf{n}$. However, in the presence of director elastic energy or boundary and topological constraints, this configuration could have a high energy cost due to possible director distortions, so that an intermediate configuration could be preferable. In such a case, it is possible that $\mathbf{n}_{*}$ and $\mathbf{n}$ do not coincide.

The minimum of the free energy, which is reached at equilibrium in an infinite domain, is yielded by

$$
\mathbf{B}_{\mathrm{e}}=\boldsymbol{\Psi}_{*}, \quad \boldsymbol{\Psi}_{*}=\boldsymbol{\Psi}, \quad \mathbf{n}=\text { constant } .
$$

In dynamics, far from equilibrium, identities (6) do not generally hold. However, when $\mu_{1} \ll \mu_{2}$ the third term in (5) dominates the second, so that it would be too energetically expensive to have two different shape tensors for long times. If the relaxation dynamics is sufficiently fast (in a sense to be specified later), we can assume to leading $\operatorname{order} \boldsymbol{\Psi}_{*}=\boldsymbol{\Psi}$ and thus recover our previous theory by considering only the second term. When $\mu_{1}$ and $\mu_{2}$ are comparable, or the relaxation dynamics is slow, we need to keep two separate shape tensors and study the dynamics that brings $\boldsymbol{\Psi}_{*}$ to evolve toward $\boldsymbol{\Psi}$ or, equivalently, $\mathbf{n}_{*}$ in the direction of $\mathbf{n}$. This dynamics is governed by an additional characteristic time $\tau_{*}$, introduced below.

\section{GOVERNING EQUATIONS}

Here, we simply state the main equations of the model. The full derivation is given in Appendix A. More details on the physical meaning of some terms may also be gathered from [15-17,19,21].

There are two types of governing equations. The first set of equations comprises balance laws that do not imply dissipation of energy. In our case, these are the equations for the velocity field $\mathbf{v}$ and the director $\mathbf{n}$ :

$$
\rho \dot{\mathbf{v}}=\mathbf{b}+\operatorname{div} \mathbf{T}, \quad \mathbf{n} \times(\mathbf{g}-\mathbf{h})=\mathbf{0},
$$

where an overdot indicates the material time derivative. The boundary conditions are

$$
\mathbf{t}_{(\boldsymbol{v})}=\mathbf{T} \boldsymbol{v}, \quad \mathbf{n} \times \mathbf{m}_{(\boldsymbol{v})}=\mathbf{n} \times\left(\rho \frac{\partial \sigma}{\partial \boldsymbol{\nabla} \mathbf{n}}\right) \boldsymbol{v} .
$$

In the above equations, $\boldsymbol{v}$ is the outer unit normal to the surface, $\mathbf{t}_{(v)}$ and $\mathbf{m}_{(v)}$ are the surface tractions and the surface couples, $\mathbf{T}$ is Cauchy stress tensor, $\mathbf{b}$ is the external body force, $\mathbf{h}$ is the nematic molecular field, and $\mathbf{g}$ is the external field acting on the director (e.g., a magnetic field) which will be be set to zero in the following for simplicity. As shown in the Appendix, $\mathbf{T}$ and $\mathbf{h}$ are defined as

$$
\begin{aligned}
\mathbf{T}:= & \rho \frac{\partial \sigma}{\partial \mathbf{F}} \mathbf{F}^{T}-\rho(\nabla \mathbf{n})^{T} \frac{\partial \sigma}{\partial \nabla \mathbf{n}} \\
& +\frac{1}{2}\left(\mathbf{h}_{*} \otimes \mathbf{n}_{*}-\mathbf{n}_{*} \otimes \mathbf{h}_{*}\right),
\end{aligned}
$$




$$
\mathbf{h}:=\rho \frac{\partial \sigma}{\partial \mathbf{n}}-\operatorname{div}\left(\rho \frac{\partial \sigma}{\partial \nabla \mathbf{n}}\right),
$$

where the molecular field $\mathbf{h}_{*}$ associated to $\mathbf{n}_{*}$ is

$$
\mathbf{h}_{*}:=\rho \frac{\partial \sigma}{\partial \mathbf{n}_{*}} .
$$

The second types of equations are associated with irreversible processes and follow from linear irreversible thermodynamics principles. These equations describe how the effective strain tensor $\mathbf{B}_{\mathrm{e}}$ and the main axis of the natural polymer network $\mathbf{n}_{*}$ evolve:

$$
\begin{gathered}
\mathbb{D}\left(\mathbf{B}_{\mathrm{e}}^{\nabla}\right)=-\frac{\partial \sigma}{\partial \mathbf{B}_{\mathrm{e}}}, \\
\lambda\left(\mathbf{n}_{*} \times \stackrel{\circ}{*}_{*}\right)=-\mathbf{n}_{*} \times \mathbf{h}_{*} .
\end{gathered}
$$

The kinematics of the material reorganization and the natural axis evolution are described by the upper-convected time derivative $\mathbf{B}_{\mathrm{e}}^{\nabla}$ and the corotational derivative of $\mathbf{n}_{*}$, defined as

$$
\begin{gathered}
\mathbf{B}_{\mathrm{e}}^{\nabla}:=\left(\mathbf{B}_{\mathrm{e}}\right)^{\cdot}-(\nabla \mathbf{v}) \mathbf{B}_{\mathrm{e}}-\mathbf{B}_{\mathrm{e}}(\nabla \mathbf{v})^{T}, \\
\stackrel{\circ}{*}_{*}:=\dot{\mathbf{n}}_{*}-\mathbf{W} \mathbf{n}_{*},
\end{gathered}
$$

where $\mathbf{W}=\frac{1}{2}\left[\nabla \mathbf{v}-(\nabla \mathbf{v})^{T}\right]$ is the spin tensor. The tensor $\mathbb{D}$ is a fourth-rank tensor which is compatible with the uniaxial symmetry about $\mathbf{n}_{*}$, has the major symmetries, and is positive definite [17], and $\lambda$ is a positive material parameter. These phenomenological quantities contain the characteristic times of material reorganization and specify what are the possible different modes of relaxation and how fast these relaxation modes drag the system to equilibrium.

For our purposes, it suffices to say that $\mathbb{D}$ comprises four relaxation times: $\tau_{1}, \tau_{2}, \tau_{3}$, and $\tau_{4}$ (see [17] for details), while $\lambda$ leads to the introduction of a fifth relaxation time $\tau_{*}$, defined in the next section. Specifically, $\tau_{1}$ measures the relaxation time of the pure shearing modes in a plane through $\mathbf{n}_{*}$ (i.e., a stretching that makes a $45^{\circ}$ angle with $\mathbf{n}_{*}$, and does not involve rotations). By contrast, $\tau_{2}$ is associated with pure sharing modes that happen in the plane orthogonal to $\mathbf{n}_{*}$.

\section{LESLIE COEFFICIENTS}

In this section we derive the explicit expressions for the Cauchy stress tensor (9), the molecular fields (10) and (11), and the relaxation Eqs. (12) and (13) when the free energy $\sigma$ is given as in Eq. (5). This will allow us to simplify our model for fast relaxation times and thus to give a physical interpretation of the Leslie coefficients in terms of our model parameters. For ease of reading, part of the calculations are reported in Appendix B.

A little algebra allows us to rearrange the Cauchy stress tensor, as given in (9) [or (A12)], in the form

$$
\begin{aligned}
& \mathbf{T}=-p \mathbf{I}+\rho \mu_{1}\left(\boldsymbol{\Psi}_{*}^{-1} \mathbf{B}_{\mathrm{e}}-\mathbf{I}\right) \\
& -\frac{\lambda}{2}\left(\stackrel{\circ}{\mathbf{n}}_{*} \otimes \mathbf{n}_{*}-\mathbf{n}_{*} \otimes \stackrel{\circ}{\mathbf{n}}_{*}\right)-\rho(\nabla \mathbf{n})^{T} \frac{\partial \sigma_{\mathrm{Fr}}}{\partial \nabla \mathbf{n}},
\end{aligned}
$$

with $p:=\rho^{2} \frac{\partial \sigma}{\partial \rho}$ a pressurelike function. The material reorganization is governed by (12), which takes the form

$$
\hat{\mathbb{D}}\left(\mathbf{B}_{\mathrm{e}}^{\nabla}\right)-\mathbf{B}_{\mathrm{e}}^{-1}+\boldsymbol{\Psi}_{*}^{-1}=\mathbf{0},
$$

where $\hat{\mathbb{D}}=2 \mathbb{D} / \mu_{1}$ is simply proportional to $\mathbb{D}$ but it has been rescaled in order to have dimensions of time. The director Eq. (7b) and the relaxation Eq. (13) are found to be (see Appendix B for details)

$$
\begin{aligned}
\operatorname{div}\left(\rho \frac{\partial \sigma_{\mathrm{Fr}}}{\partial \nabla \mathbf{n}}\right)+\rho \mu_{2} & \frac{\left(a^{3}-1\right)^{2}}{a^{3}}\left(\mathbf{n}_{*} \cdot \mathbf{n}\right)\left(\mathbf{n} \times \mathbf{n}_{*}\right)=0, \\
\tau_{*}\left(\mathbf{n}_{*} \times \stackrel{\circ}{\mathbf{n}}_{*}\right)= & \frac{\mu_{1}}{\mu_{2}} \frac{a^{3}}{a^{3}-1}\left(\mathbf{n}_{*} \times \mathbf{B}_{\mathrm{e}} \mathbf{n}_{*}\right) \\
& +\left(\mathbf{n}_{*} \cdot \mathbf{n}\right)\left(\mathbf{n}_{*} \times \mathbf{n}\right),
\end{aligned}
$$

where

$$
\tau_{*}=\frac{a^{3}}{\left(a^{3}-1\right)^{2}} \frac{\lambda}{\rho \mu_{2}}
$$

is proportional to the parameter $\lambda$ and can be taken as the characteristic time associated with the reorientation of $\mathbf{n}_{*}$. At the end of Sec. III we have seen that when the material undergoes a pure shear strain in a plane through $\mathbf{n}_{*}$, the effective strain tensor relaxes to the natural state with a characteristic time $\tau_{1}$. An alternative mechanism to relax the internal stress is to rotate the unit cell of the natural network (and its main axis $\mathbf{n}_{*}$ ) in order to conform to a general superimposed deformation or in response to a mismatch with the director field $\mathbf{n}$, and this happens with a characteristic time $\tau_{*}$.

It is easy to see that $\tau_{1}$ and $\tau_{*}$ are independent times and indeed can be very different. Let us consider a nematic elastomer. Its rubbery network is elastic and does not reorganize, so that $\tau_{1}$ can be considered infinite. By contrast, $\tau_{*}$ is finite and is interpreted as the time that the director $\mathbf{n}$ takes to coincide with the principal direction of the superimposed strain (for NEs $\mathbf{n}=\mathbf{n}_{*}$ by assumption).

It is interesting to observe that, according to Eq. (18), when the director field is homogeneous, i.e., $|\nabla \mathbf{n}|=\mathbf{0}, \mathbf{n}_{*}$ is either parallel or orthogonal to $\mathbf{n}$. This can also be seen from the energy density. Whenever the Frank potential vanishes in (5), every possible configuration of minimum energy satisfies $\boldsymbol{\Psi}_{*}=\boldsymbol{\Psi}$ or, in other terms, $\mathbf{n}_{*}=\mathbf{n}$. Any deviation from $\mathbf{n}_{*}=$ $\mathbf{n}$ costs some energy and this excess energy is ultimately due to distortions in the director field.

When $\mu_{1} \ll \mu_{2}$ and we assume that $\tau_{i}(i=1,2,3,4)$ and $\tau_{*}$ are much smaller that the characteristic times associated with the deformation, measured by $\tau_{\text {def }}=\max \{1 /|\nabla \mathbf{v}|\}, \mathbf{B}_{\mathrm{e}}$ and $\mathbf{n}_{*}$ are just small corrections of their equilibrium values $\boldsymbol{\Psi}_{*}$ and $\mathbf{n}$. Equation (17) then yields the approximation of $\mathbf{B}_{\mathrm{e}}$ to first order

$$
\mathbf{B}_{\mathrm{e}} \approx \boldsymbol{\Psi}_{*}-\boldsymbol{\Psi}_{*} \hat{\mathbb{D}}\left(\boldsymbol{\Psi}_{*}^{\nabla}\right) \boldsymbol{\Psi}_{*} .
$$

This approximation is suitable for the description of a fluidlike behavior and, therefore, it is appropriate for NLCs and possibly for some nematic polymers (when viscoelastic effects are not important), but it is not applicable to the other possible extreme of the model, namely, nematic elastomers.

To obtain the approximation of the stress tensor (16) to first order, it is sufficient to consider only the leading term of (19). 
To leading order, $\mathbf{n}_{*} \approx \mathbf{n}$, so that (16) becomes

$$
\begin{aligned}
& \mathbf{T}=-p \mathbf{I}-\rho \mu_{1} \hat{\mathbb{D}}(\boldsymbol{\Psi} \nabla) \boldsymbol{\Psi}
\end{aligned}
$$

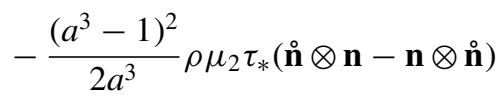

$$
\begin{aligned}
& -\rho(\nabla \mathbf{n})^{T} \frac{\partial \sigma_{\mathrm{Fr}}}{\partial \nabla \mathbf{n}} .
\end{aligned}
$$

If we now compare (22) with the classical expression of the Cauchy stress tensor, as given by the compressible EricksenLeslie theory

$$
\begin{aligned}
& \mathbf{T}=-p \mathbf{I}+\alpha_{1}(\mathbf{n} \cdot \mathbf{D n})(\mathbf{n} \otimes \mathbf{n})+\alpha_{2}(\stackrel{\circ}{\mathbf{n}} \otimes \mathbf{n})+\alpha_{3}(\mathbf{n} \otimes \stackrel{\mathbf{n}}{ }) \\
& +\alpha_{4} \mathbf{D}+\alpha_{5}(\mathbf{D n} \otimes \mathbf{n})+\alpha_{6}(\mathbf{n} \otimes \mathbf{D n}) \\
& +\alpha_{7}[(\operatorname{tr} \mathbf{D})(\mathbf{n} \otimes \mathbf{n})+(\mathbf{n} \cdot \mathbf{D n}) \mathbf{I}]+\alpha_{8}(\operatorname{tr} \mathbf{D}) \mathbf{I},
\end{aligned}
$$

and use the explicit expression for $\hat{\mathbb{D}}$ as given in Ref. [17], we obtain that the Leslie coefficients in terms of our model parameters are

$$
\begin{aligned}
\alpha_{1}= & \rho \mu_{1}(\rho)\left(\tau_{2}-\frac{\left[a(\rho)^{3}+1\right]^{2}}{a(\rho)^{3}} \tau_{1}+3 \tau_{3}(\cos \Theta)^{2}\right. \\
& \left.+3 \tau_{4}(\sin \Theta)^{2}\right), \\
\alpha_{2}= & -\rho \mu_{1}(\rho) \tau_{1}\left[a(\rho)^{3}-1\right]-\rho \mu_{2}(\rho) \tau_{*} \frac{\left[a(\rho)^{3}-1\right]^{2}}{2 a(\rho)^{3}} \\
\alpha_{3}= & -\rho \mu_{1}(\rho) \tau_{1}\left[1-a(\rho)^{-3}\right]+\rho \mu_{2}(\rho) \tau_{*} \frac{\left[a(\rho)^{3}-1\right]^{2}}{2 a(\rho)^{3}} \\
\alpha_{4}= & 2 \rho \mu_{1}(\rho) \tau_{2}, \\
\alpha_{5}= & \rho \mu_{1}(\rho)\left(\left[1+a(\rho)^{3}\right] \tau_{1}-2 \tau_{2}\right), \\
\alpha_{6}= & \rho \mu_{1}(\rho)\left(\left[1+a(\rho)^{-3}\right] \tau_{1}-2 \tau_{2}\right),
\end{aligned}
$$

where $\Theta$ is an additional parameter that appears in the definition of $\mathbb{D}$ and affects $\alpha_{1}$ but plays no role in what follows. It is also possible to find the bulk viscosity coefficients $\alpha_{7}$ and $\alpha_{8}$, but these are not particularly relevant for the purposes of this paper, and we omit them for brevity.

It is interesting to observe that, in agreement with experiments, $\alpha_{2}$ is always negative for rodlike LCs $[a(\rho)>1]$ as it is obtained as the sum of two negative terms. By contrast, $\alpha_{3}$ can be either negative or positive, the latter case leading to a tumbling behavior. Vice versa, for disklike molecules $\alpha_{3}$ is always positive while $\alpha_{2}$ can be positive (flow alignment) or negative (tumbling).

The Parodi relation is automatically satisfied along with a second identity [16]

$$
\alpha_{6}-\alpha_{5}=\alpha_{2}+\alpha_{3}, \quad \frac{\alpha_{4}+\alpha_{5}}{\alpha_{4}+\alpha_{6}}=\frac{\alpha_{2}+\lambda / 2}{\alpha_{3}-\lambda / 2}=a(\rho)^{3},
$$

where, we recall, $\lambda=\rho \mu_{2} \tau_{*}\left(a^{3}-1\right)^{2} / a^{3}$.

\section{TUMBLING PARAMETER}

In terms of Leslie coefficients, it is known that a tumbling instability arises whenever $\alpha_{3} / \alpha_{2}<0$ [8,20], a condition that,

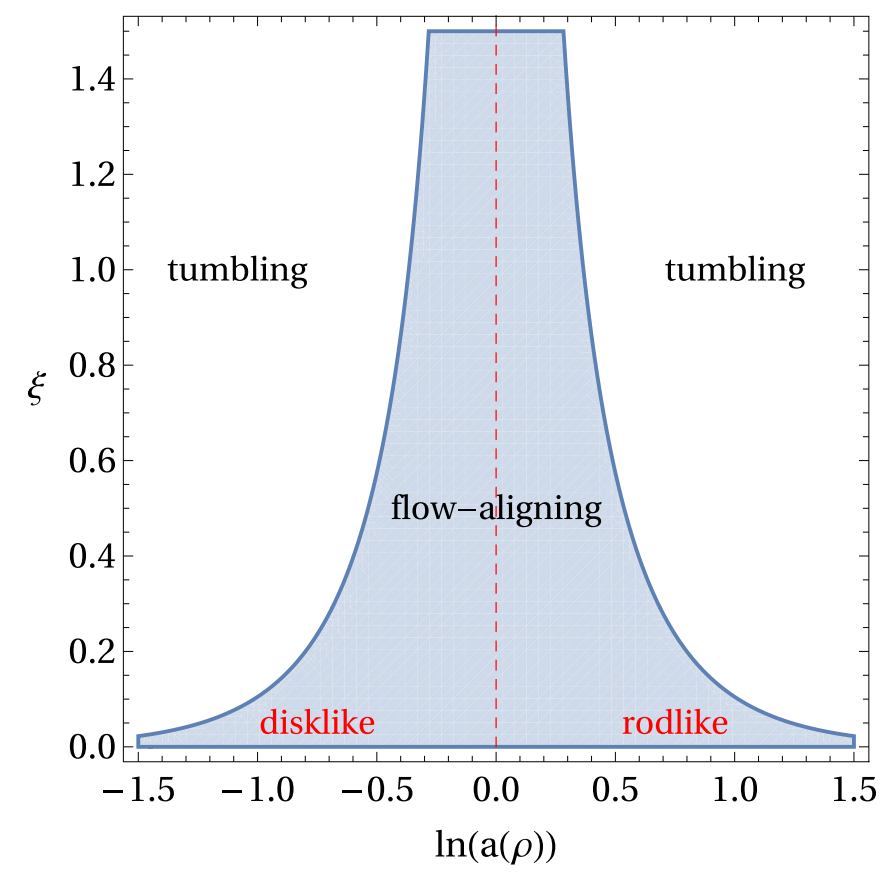

FIG. 2. Flow-aligning (blue) and tumbling (white) regions, as deduced from (28), as a function of the model parameters $a(\rho)$ and $\xi$. The region with $a(\rho)>1$ corresponds to prolate shape tensors, associated with rodlike molecules. Disklike molecules correspond to $a(\rho)<1$. Tumbling ceases to exist in the isotropic phase, where the shape tensors are spherical $[a(\rho)=1]$.

after the substitution of (23), reads as

$$
\frac{\alpha_{3}}{\alpha_{2}}=\frac{1}{a(\rho)^{3}}\left(\frac{2-\xi\left[a(\rho)^{3}-1\right]}{2-\xi\left[a(\rho)^{-3}-1\right]}\right)<0,
$$

where we have defined the key ratio

$$
\xi=\frac{\mu_{2} \tau_{*}}{\mu_{1} \tau_{1}} .
$$

In fact, the flow alignment angle is known to be

$$
\tan \theta=\sqrt{\frac{\alpha_{3}}{\alpha_{2}}},
$$

so that alignment is only possible when $\alpha_{2}$ and $\alpha_{3}$ have the same sign (both positive or negative). If $\xi \approx 0$, so that the natural network axis is free to reorient with the flow, only flow alignment is possible. By simplifying (25), we get the following condition for tumbling behavior:

$$
\begin{array}{lll}
\xi>\frac{2}{a(\rho)^{-3}-1} \quad \text { if } & 0<a(\rho)<1, \\
\xi>\frac{2}{a(\rho)^{3}-1} \quad \text { if } & a(\rho)>1 .
\end{array}
$$

In either case, tumbling occurs when the ratio $\frac{\mu_{2} \tau_{*}}{\mu_{1} \tau_{1}}$ is larger than a given threshold, which depends on the anisotropy of the shape tensor [i.e., on the aspect ratio $a(\rho)]$. This threshold goes to infinity in the isotropic case $a(\rho)=1$ and decreases for increasing anisotropy of the shape tensor (see Fig. 2). Even if our theory does not depend on temperature, it is reasonable to expect that in the isotropic phase the shape tensor becomes spherical, i.e., $a(\rho)=1$. Hence, we get a clear explanation 
of why tumbling is enhanced by the presence of orientational order, and it is suppressed in the isotropic phase, even for nematic polymers.

We have seen that $\xi$ represents the ratio between two possible effects: relaxation by alignment of the natural network axis with the flow, and strain relaxation by material reorganization. As is clear from Fig. 2, if the first mechanism prevails, the material flow aligns, while if the second is more efficient, the director tumbles (when sufficiently far from the isotropic phase). This fact is more pronounced for long chains or flat disks, i.e., it increases with molecular anisotropy.

This interpretation is in agreement with previous experiments and with some earlier theoretical claims, where tumbling was explained in terms of strong side-to-side molecular association [6,7,22]. In particular, the authors of Ref. [22] observed experimentally that "addition of a side-chain LCP (liquid crystal polymer) to flow-aligning 5CB induces a directortumbling response, whereas dissolution of a main-chain LCP in director-tumbling $8 \mathrm{CB}$ induces a flow-aligning response." This agrees with our interpretation in that strong side-chain association may hinder natural network rotations while it does not have much influence on the material reorganization by network subcell sliding.

A more direct way to reach the same conclusions, that does not make use of the Leslie coefficients, is to study the evolution of the natural network main axis using Eq. (19). In the absence of nematic distortions, from the balance equation (18), we get that $\mathbf{n}=\mathbf{n}_{*}$. Hence, in the limit $\mu_{2} \tau_{*} \ll \mu_{1} \tau_{1}$, Eq. (19) to leading order reads as $\mathbf{n}_{*} \times \mathbf{B}_{\mathrm{e}} \mathbf{n}_{*}=0$, i.e., $\mathbf{n}_{*}$ aligns with a principal direction of the effective strain (which is fixed in the fast relaxation approximation discussed above). In this case, $\mathbf{n}_{*}$ is constant and aligns with the flow. On the other hand, if $\mu_{2} \tau_{*} \gg \mu_{1} \tau_{1}$, to leading order we have $\mathbf{n}_{*} \times \grave{\mathbf{n}}_{*}=0$. Since $\stackrel{\mathbf{n}}{*}_{*}$ is orthogonal to $\mathbf{n}_{*}$, this implies that $\stackrel{\mathbf{n}}{*}_{*}$ must vanish. In such a case, we do not obtain a steady solution, but a rotating field: $\dot{\mathbf{n}}_{*}=\mathbf{W} \mathbf{n}_{*}$.

More precisely, let us consider a simple shear flow in a semi-infinite medium, under the usual assumption of fast relaxation approximation $\left(\mu_{2} \gg \mu_{1}\right.$ and $\left.\tau_{*}, \tau_{i} \ll \tau_{\text {def }}\right)$. The velocity field is written as $\mathbf{v}=\dot{\gamma} y \mathbf{e}_{x}$, where $\dot{\gamma}$ is the shear rate and $\mathbf{e}_{x}$ is a unit vector along the $x$ axis. Within our approximation, $\mathbf{n}_{*}$ is just a small perturbation of its equilibrium value $\mathbf{n}$ and we posit

$$
\varepsilon=\mu_{1} / \mu_{2}, \quad \mathbf{n}_{*}=\mathbf{n}+\mathbf{n}_{1}, \quad \mathbf{n}_{1}=O(\varepsilon),
$$

where $\mathbf{n}$ is taken to be homogeneous over the whole sample (i.e., $|\nabla \mathbf{n}|=0$ ), $\mathbf{n}_{1} \cdot \mathbf{n}=0$, and $\mathbf{n}_{1}$ measures the difference between $\mathbf{n}_{*}$ and $\mathbf{n}$ in a dynamic situation. The relaxation times are much smaller than $\tau_{\mathrm{def}}$, so that the product of either $\tau_{*}$ or $\tau_{1}$ with a time derivative (i.e., $\mathbf{n}$ or $\mathbf{D n}$ ) is taken to be $O(\varepsilon)$. For a homogeneous director field, the balance Eq. (18) implies that $\mathbf{n}_{1}$ vanishes to first order, so that we can assume $\mathbf{n}_{*} \approx \mathbf{n}$. Hence, Eq. (19) reads as

$$
\tau_{*}(\mathbf{n} \times \stackrel{\circ}{\mathbf{n}})=-\frac{\mu_{1}}{\mu_{2}} \frac{a^{3}}{a^{3}-1}\left[\mathbf{n} \times \boldsymbol{\Psi} \hat{\mathbb{D}}\left(\Psi^{\nabla}\right) \boldsymbol{\Psi} \mathbf{n}\right] .
$$

The codeformational derivative of $\boldsymbol{\Psi}$ can be explicitly calculated in terms of the imposed macroscopic flow so that
Eq. (30) simplifies to

$$
\xi\left(\mathbf{n} \times \stackrel{\mathbf{n}}{)}=-\mathbf{n} \times\left(\stackrel{\circ}{\mathbf{n}}-\frac{a^{3}+1}{a^{3}-1} \mathbf{D n}\right) .\right.
$$

We now introduce the tilt angle $\theta$ such that $\mathbf{n}=\sin \theta(t) \mathbf{e}_{x}+$ $\cos \theta(t) \mathbf{e}_{y}$. After a little algebra, (31) can be written as

$$
(1+\xi) \dot{\theta}=\frac{\dot{\gamma}}{2}\left(1+\xi+\frac{a^{3}+1}{a^{3}-1} \cos (2 \theta)\right) .
$$

When the liquid crystal aligns with the macroscopic flow, the angle $\theta$ is constant, so that $\dot{\theta}=0$. Thus, stationary solutions are only possible if

$$
\frac{\left|a^{3}-1\right|}{a^{3}+1} \leqslant \frac{1}{1+\xi}
$$

a condition that, after some simplification, is shown to coincide with the flow-aligning condition $\alpha_{3} / \alpha_{2} \geqslant 0$, as derived from Leslie coefficients (23), and is complementary to the tumbling condition (28). When the condition (33) is not met [or, equivalently, the complementary condition (28) holds], we can still solve (32) in this regime to obtain the periodic oscillations of the director, i.e., the functional dependence of $\theta$ over time.

\section{CONCLUSIONS}

The mechanisms underlying tumbling instability are subtle and there is no widely accepted explanation for the physical origins of this phenomenon. We find that the distinction between the nematic director and the principal axis of the natural polymer network is the key feature that allows us to observe the crossover between flow-aligning and tumbling behaviors.

This distinction allows the material, when it undergoes a shearing deformation, to relax the internal stress in two distinct ways. The first is the internal reorganization of the polymer network cross links, the second is the rotation of the natural polymer network main axis to align with the principal direction of the effective strain. Both these mechanisms reduce the internal stress, but tumbling occurs whenever the first mechanism prevails over the second.

In agreement with previous claims, this explanation suggests that tumbling is due to a strong side-to-side molecular association, either by electrostatic interactions or by steric interaction (for example, in long flexible polymer chains). In our model, a single material parameter $\xi$, defined as the ratio $\mu_{2} \tau_{*} / \mu_{1} \tau_{1}$, describes the relative importance of one mechanism over the other. Furthermore, we show that in the isotropic phase only flow aligning is possible and that tumbling is enhanced by strong molecular anisotropy.

For simplicity, we have developed a minimal model. Our analysis does not include all possible scenarios that are believed to play an important role in the current understanding of tumbling in nematic fluids. For instance, we do not explore lyotropic materials and concentration dependence, temperature effects, compounds of different molecules. In particular, in order to fully study the tumbling dependence on the degree of order and the temperature effects, it is necessary to construct a theory that includes the nematic ordering tensor $\mathbf{Q}$. 
It is expected that the resulting theory in this case be highly nontrivial and its analysis is postponed to a following paper.

Another important reason for introducing the tensor $\mathbf{Q}$ in our model is that tumbling typically generates defects [23], indicating that the system could no longer be regarded as a monodomain [3]. It is observed in [23] that the defect structures, or textures, in a 8CB sample depend on the shear history of the sample. In particular, the texture depends not only on the rotation speed, but also on the rate at which the rotation speed is increased from zero. This is in agreement with the viscoelastic nature of our model and the consequent interpretation of tumbling in terms of relaxation processes. By contrast, the Ericksen-Leslie model, having frequencyindependent viscosity coefficients, cannot reproduce different material behaviors or aligning features for different shear rates or shear histories.

\section{ACKNOWLEDGMENT}

I would like to thank A. Di Carlo and P. Biscari for their valuable and helpful suggestions during the planning and development of this research.

\section{APPENDIX A: DERIVATION OF THE MODEL}

In this Appendix, we derive the governing equations. The second principle of thermodynamics requires that, for any isothermal process, for any portion $\mathcal{P}_{t}$ of the body at all times, the dissipation (rate of entropy production) be greater or equal than zero [24]:

$$
\mathcal{D}:=W^{(\text {ext })}-\dot{K}-\dot{\mathcal{F}} \geqslant 0
$$

where $W^{(\mathrm{ext})}$ is the power expended by the external forces, $\dot{K}$ is the rate of change of the kinetic energy, $\dot{\mathcal{F}}$ is the rate of change of the free energy, and the dissipation $\mathcal{D}$ is a positive quantity that represents the energy loss due to irreversible process. Here, an overdot indicates the material time derivative. More precisely, we define

$$
\begin{aligned}
& W^{(\mathrm{ext})}=\int_{\mathcal{P}_{t}} \mathbf{b} \cdot \mathbf{v} d v+\int_{\partial \mathcal{P}_{t}} \mathbf{t}_{(\boldsymbol{v})} \cdot \mathbf{v} d a \\
& +\int_{\mathcal{P}_{t}} \mathbf{g} \cdot \dot{\mathbf{n}} d v+\int_{\partial \mathcal{P}_{t}} \mathbf{m}_{(v)} \cdot \dot{\mathbf{n}} d a, \\
& K+\mathcal{F}=\int_{\mathcal{P}_{t}}\left(\frac{1}{2} \rho \mathbf{v}^{2}+\rho \sigma\left(\rho, \mathbf{B}_{\mathrm{e}}, \mathbf{n}_{*}, \mathbf{n}, \nabla \mathbf{n}\right)\right) d v \\
& \mathcal{D}=\int_{\mathcal{P}_{t}} \xi d v, \quad \xi \geqslant 0 .
\end{aligned}
$$

The unit vector $v$ is the external unit normal to the boundary $\partial \mathcal{P}_{t} ; \mathbf{b}$ is the external body force, $\mathbf{t}_{(v)}$ is the external traction on the bounding surface $\partial \mathcal{P}_{t}$. The vector fields $\mathbf{g}$ and $\mathbf{m}_{(v)}$ are the external generalized forces conjugate to the microstructure: $\mathbf{n} \times \mathbf{g}$ is usually interpreted as "external body moment" and $\mathbf{n} \times \mathbf{m}_{(\boldsymbol{v})}$ is interpreted as "surface moment per unit area" (the couple stress vector).
The material time derivative of $\mathcal{F}$ is

$$
\begin{aligned}
\dot{\mathcal{F}}= & \int_{\mathcal{P}_{t}}\left(\rho \frac{\partial \sigma}{\partial \mathbf{F}} \cdot \dot{\mathbf{F}}+\rho \frac{\partial \sigma}{\partial \mathbf{H}} \cdot \dot{\mathbf{H}}\right. \\
& \left.+\rho \frac{\partial \sigma}{\partial \mathbf{n}} \cdot \dot{\mathbf{n}}+\rho \frac{\partial \sigma}{\partial \mathbf{n}_{*}} \cdot \dot{\mathbf{n}}_{*}+\rho \frac{\partial \sigma}{\partial \nabla \mathbf{n}} \cdot(\nabla \mathbf{n})\right) d v .
\end{aligned}
$$

If we introduce the (frame-indifferent) upper-convected time derivative $\mathbf{B}_{\mathrm{e}}^{\nabla}$, as given in Eq. (14), and use the identities

$$
\begin{gathered}
\dot{\mathbf{F}}=(\nabla \mathbf{v}) \mathbf{F}, \\
(\nabla \mathbf{n})^{\cdot}=\nabla \dot{\mathbf{n}}-(\nabla \mathbf{n})(\nabla \mathbf{v}), \\
\frac{\partial \sigma}{\partial \mathbf{B}_{\mathrm{e}}}=\mathbf{F}^{-T} \frac{\partial \sigma}{\partial \mathbf{H}} \mathbf{F}^{-1}, \\
\mathbf{B}_{\mathrm{e}}^{\nabla}=\dot{F H}^{T},
\end{gathered}
$$

Eq. (A5) simplifies to

$$
\begin{aligned}
\dot{\mathcal{F}}= & \int_{\mathcal{P}_{t}}\left(\rho \frac{\partial \sigma}{\partial \mathbf{F}} \mathbf{F}^{T} \cdot \nabla \mathbf{v}+\rho \frac{\partial \sigma}{\partial \mathbf{B}_{\mathrm{e}}} \cdot \mathbf{B}_{\mathrm{e}}^{\nabla}+\rho \frac{\partial \sigma}{\partial \mathbf{n}} \cdot \dot{\mathbf{n}}\right. \\
& \left.+\rho \frac{\partial \sigma}{\partial \mathbf{n}_{*}} \cdot \dot{\mathbf{n}}_{*}+\rho \frac{\partial \sigma}{\partial \nabla \mathbf{n}} \cdot(\nabla \mathbf{n})\right) d v \\
= & \int_{\mathcal{P}_{t}}\left(\rho \frac{\partial \sigma}{\partial \mathbf{F}} \mathbf{F}^{T}-\rho(\nabla \mathbf{n})^{T} \frac{\partial \sigma}{\partial \nabla \mathbf{n}}\right) \cdot \nabla \mathbf{v} d v \\
& +\int_{\mathcal{P}_{t}}\left(\rho \frac{\partial \sigma}{\partial \mathbf{n}} \cdot \dot{\mathbf{n}}+\rho \frac{\partial \sigma}{\partial \nabla \mathbf{n}} \cdot \nabla \dot{\mathbf{n}}\right) d v \\
& +\int_{\mathcal{P}_{t}}\left(\rho \frac{\partial \sigma}{\partial \mathbf{n}_{*}} \cdot \dot{\mathbf{n}}_{*}\right) d v+\int_{\mathcal{P}_{t}} \rho \frac{\partial \sigma}{\partial \mathbf{B}_{\mathrm{e}}} \cdot \mathbf{B}_{\mathrm{e}}^{\nabla} d v .
\end{aligned}
$$

We now define the molecular fields $\mathbf{h}$ and $\mathbf{h}_{*}$, as in Eqs. (10) and (11), so that we rewrite the second integral as

$$
\begin{aligned}
\int_{\mathcal{P}_{t}} & \left(\rho \frac{\partial \sigma}{\partial \mathbf{n}} \cdot \dot{\mathbf{n}}+\rho \frac{\partial \sigma}{\partial \boldsymbol{\nabla} \mathbf{n}} \cdot \nabla \dot{\mathbf{n}}\right) d v \\
= & \int_{\partial \mathcal{P}_{t}}\left(\rho \frac{\partial \sigma}{\partial \nabla \mathbf{n}}\right) \boldsymbol{v} \cdot \dot{\mathbf{n}} d a+\int_{\mathcal{P}_{t}} \mathbf{h} \cdot \dot{\mathbf{n}} d v .
\end{aligned}
$$

Furthermore, since we assume that a realignment of the relaxed network axis with the flow gives a positive contribution to the dissipation, we have to recast the second-last integral in (A10) in terms of frame-indifferent fields (a vanishing dissipation is associated with a rigid rotation of the whole body). Hence, we write

$$
\begin{aligned}
\int_{\mathcal{P}_{t}} \mathbf{h}_{*} \cdot \dot{\mathbf{n}}_{*} d v= & \int_{\mathcal{P}_{t}} \mathbf{h}_{*} \cdot \stackrel{\mathbf{n}}{*}_{*} d v+\int_{\mathcal{P}_{t}} \mathbf{h}_{*} \cdot \mathbf{W} \mathbf{n}_{*} d v \\
= & \int_{\mathcal{P}_{t}} \mathbf{h}_{*} \cdot \stackrel{\circ}{*}_{*} d v+\int_{\mathcal{P}_{t}} \frac{1}{2}\left(\mathbf{h}_{*} \otimes \mathbf{n}_{*}\right. \\
& \left.-\mathbf{n}_{*} \otimes \mathbf{h}_{*}\right) \cdot \nabla \mathbf{v} d v
\end{aligned}
$$

The last term is paired with $\nabla \mathbf{v}$ so that it represents a contribution to the Cauchy stress tensor, defined as in Eq. (9) and 
repeated here for convenience:

$$
\mathbf{T}:=\rho \frac{\partial \sigma}{\partial \mathbf{F}} \mathbf{F}^{T}-\rho(\nabla \mathbf{n})^{T} \frac{\partial \sigma}{\partial \nabla \mathbf{n}}+\frac{1}{2}\left(\mathbf{h}_{*} \otimes \mathbf{n}_{*}-\mathbf{n}_{*} \otimes \mathbf{h}_{*}\right) .
$$

Therefore, the final expression for the rate of change of the free energy is

$$
\begin{aligned}
\dot{\mathcal{F}}= & \int_{\mathcal{P}_{t}} \mathbf{T} \cdot \nabla \mathbf{v} d v+\int_{\partial \mathcal{P}_{t}}\left(\rho \frac{\partial \sigma}{\partial \nabla \mathbf{n}}\right) \mathbf{v} \cdot \dot{\mathbf{n}} d a \\
& +\int_{\mathcal{P}_{t}} \mathbf{h} \cdot \dot{\mathbf{n}} d v+\int_{\mathcal{P}_{t}} \mathbf{h}_{*} \cdot \stackrel{\mathbf{n}}{*}_{*} d v+\int_{\mathcal{P}_{t}} \rho \frac{\partial \sigma}{\partial \mathbf{B}_{\mathrm{e}}} \cdot \mathbf{B}_{\mathrm{e}}^{\nabla} d v \\
= & \int_{\partial \mathcal{P}_{t}} \mathbf{T} \boldsymbol{v} \cdot \mathbf{v} d a-\int_{\mathcal{P}_{t}} \operatorname{div} \mathbf{T} \cdot \mathbf{v} d v \\
& +\int_{\partial \mathcal{P}_{t}}\left(\rho \frac{\partial \sigma}{\partial \nabla \mathbf{n}}\right) \boldsymbol{v} \cdot \dot{\mathbf{n}} d a+\int_{\mathcal{P}_{t}} \mathbf{h} \cdot \dot{\mathbf{n}} d v \\
& +\int_{\mathcal{P}_{t}} \mathbf{h}_{*} \cdot \stackrel{\mathbf{n}}{*}_{*} d v+\int_{\mathcal{P}_{t}} \rho \frac{\partial \sigma}{\partial \mathbf{B}_{\mathrm{e}}} \cdot \mathbf{B}_{\mathrm{e}}^{\nabla} d v,
\end{aligned}
$$

and the dissipation is then written as

$$
\begin{aligned}
\mathcal{D}= & W^{(\mathrm{ext})}-\dot{K}-\dot{\mathcal{F}} \\
= & \int_{\mathcal{P}_{t}}(\mathbf{b}-\rho \dot{\mathbf{v}}+\operatorname{div} \mathbf{T}) \cdot \mathbf{v} d v+\int_{\partial \mathcal{P}_{t}}\left(\mathbf{t}_{(\boldsymbol{v})}-\mathbf{T} \boldsymbol{v}\right) \cdot \mathbf{v} d a \\
& +\int_{\mathcal{P}_{t}}(\mathbf{g}-\mathbf{h}) \cdot \dot{\mathbf{n}} d v+\int_{\partial \mathcal{P}_{t}}\left[\mathbf{m}_{(v)}-\left(\rho \frac{\partial \sigma}{\partial \nabla \mathbf{n}}\right) \mathbf{v}\right] \cdot \dot{\mathbf{n}} d a \\
& -\int_{\mathcal{P}_{t}} \mathbf{h}_{*} \cdot \stackrel{\mathbf{n}}{*}_{*} d v-\int_{\mathcal{P}_{t}} \rho \frac{\partial \sigma}{\partial \mathbf{B}_{\mathrm{e}}} \cdot \mathbf{B}_{\mathrm{e}}^{\nabla} d v .
\end{aligned}
$$

By assumption, a positive dissipation is associated to material reorganization and only the last two integrals can contribute to the irreversible processes (i.e., can have a positive dissipation). Thus, the contribution from the first integrals must vanish and we have the equations (we recall that $\mathbf{n} \cdot \dot{\mathbf{n}}=$ 0 ) for the deformation field $\mathbf{v}$ and the director field $\mathbf{n}$, as given in Eqs. (7). The dissipation then simplifies to

$$
\mathcal{D}=-\int_{\mathcal{P}_{t}}\left(\mathbf{h}_{*} \cdot \grave{\mathbf{n}}_{*}+\rho \frac{\partial \sigma}{\partial \mathbf{B}_{\mathrm{e}}} \cdot \mathbf{B}_{\mathrm{e}}^{\nabla}\right) d v .
$$

A simple choice that satisfies $\mathcal{D} \geqslant 0$ at all times and is consistent with standard linear irreversible thermodynamics $[20,25]$ is to take the fluxes proportional to forces. In so doing, we arrive at Eqs. (12) and (13). Furthermore, we assume Onsager reciprocal relations, so that the proportionality coefficient $\mathbb{D}$ is a fourth-rank tensor which is compatible with the uniaxial symmetry about $\mathbf{n}_{*}$, has the major symmetries and is positive definite [i.e., such that $\mathbb{D}(\mathbf{A}) \cdot \mathbf{A}>0, \forall \mathbf{A} \neq 0$ and symmetric], while $\lambda$ is a positive material parameter. Indeed, it can be shown that only one coefficient $\lambda$ is necessary in (13) for symmetry reasons (see [20]). Equation (13) governs the dynamics that brings $\mathbf{n}_{*}$ toward $\mathbf{n}$ (or vice versa) and the parameter $\lambda$ contains the characteristic time of this relaxation process.

Finally, we note that, if we denote by $\mathbf{W}_{\mathbf{a}}$ the skewsymmetric tensor with axial vector a, we have from (13)

$$
\begin{aligned}
& \frac{1}{2}\left(\mathbf{h}_{*} \otimes \mathbf{n}_{*}-\mathbf{n}_{*} \otimes \mathbf{h}_{*}\right)=\frac{1}{2} \mathbf{W}_{\mathbf{n}_{*} \times \mathbf{h}_{*}} \\
& \quad=-\frac{\lambda}{2} \mathbf{W}_{\mathbf{n}_{*} \times \mathbf{n}_{*}}=-\frac{\lambda}{2}\left(\stackrel{\circ}{\mathbf{n}}_{*} \otimes \mathbf{n}_{*}-\mathbf{n}_{*} \otimes \stackrel{\circ}{\mathbf{n}}_{*}\right),
\end{aligned}
$$

so that the Cauchy stress tensor can also be written in a more familiar form as

$$
\mathbf{T}=\rho \frac{\partial \sigma}{\partial \mathbf{F}} \mathbf{F}^{T}-\rho(\nabla \mathbf{n})^{T} \frac{\partial \sigma}{\partial \nabla \mathbf{n}}-\frac{\lambda}{2}\left(\stackrel{\mathbf{n}}{*}_{*} \otimes \mathbf{n}_{*}-\mathbf{n}_{*} \otimes \stackrel{\mathbf{n}}{*}\right) .
$$

\section{APPENDIX B: DERIVATION OF THE LESLIE COEFFICIENTS}

In order to find the director equation and the the relaxation equations, we need to explicitly elaborate on the terms

$$
\begin{aligned}
& \frac{\partial \sigma}{\partial \mathbf{B}_{\mathrm{e}}}=\frac{1}{2} \mu_{1}\left(\boldsymbol{\Psi}_{*}^{-1}-\mathbf{B}_{\mathrm{e}}^{-1}\right), \\
& \frac{\partial \sigma}{\partial \mathbf{n}}=2\left(a^{2}-a^{-1}\right) \frac{\partial \sigma}{\partial \Psi} \mathbf{n} \\
& =\mu_{2}\left(a^{2}-a^{-1}\right) \boldsymbol{\Psi}_{*}^{-1} \mathbf{n}, \\
& \frac{\partial \sigma}{\partial \mathbf{n}_{*}}=2\left(a^{-2}-a\right) \frac{\partial \sigma}{\partial\left(\Psi_{*}^{-1}\right)} \mathbf{n}_{*} \\
& =\left(a^{-2}-a\right)\left(\mu_{1} \mathbf{B}_{\mathrm{e}}+\mu_{2} \boldsymbol{\Psi}\right) \mathbf{n}_{*}, \\
& \mathbf{n} \times \mathbf{h}=-\rho \mu_{2} \frac{\left(a^{3}-1\right)^{2}}{a^{3}}\left(\mathbf{n}_{*} \cdot \mathbf{n}\right)\left(\mathbf{n} \times \mathbf{n}_{*}\right) \\
& -\operatorname{div}\left(\rho \frac{\partial \sigma_{\mathrm{Fr}}}{\partial \boldsymbol{\nabla n}}\right), \\
& \mathbf{n}_{*} \times \mathbf{h}_{*}=\rho \mu_{1}\left(a^{-2}-a\right) \mathbf{n}_{*} \times \mathbf{B}_{\mathrm{e}} \mathbf{n}_{*} \\
& -\rho \mu_{2} \frac{\left(a^{3}-1\right)^{2}}{a^{3}}\left(\mathbf{n}_{*} \cdot \mathbf{n}\right)\left(\mathbf{n}_{*} \times \mathbf{n}\right) .
\end{aligned}
$$

[1] R. D. Kamien, Phys. Rev. E 61, 2888 (2000).

[2] R. Larson and L. Archer, Liq. Cryst. 19, 883 (1995).

[3] J. F. Fatriansyah and H. Orihara, Phys. Rev. E 88, 012510 (2013).

[4] A. Zakharov, A. Vakulenko, and J. Thoen, J. Chem. Phys. 118, 4253 (2003).

[5] I. Quijada-Garrido, H. Siebert, P. Becker, C. Friedrich, and C. Schmidt, Rheol. Acta 38, 495 (1999).

[6] C. Gähwiller, Phys. Rev. Lett. 28, 1554 (1972).
[7] L. Archer and R. Larson, J. Chem. Phys. 103, 3108 (1995).

[8] C. J. Chan and E. M. Terentjev, in Modeling of Soft Matter, edited by M.-C. T. Calderer and E. M. Terentjev (Springer, New York, 2005), pp. 27-84.

[9] M. Doi, J. Polym. Sci.: Polym. Phys. Edition 19, 229 (1981).

[10] N. Kuzuu and M. Doi, J. Phys. Soc. Jpn. 52, 3486 (1983).

[11] N. Kuzuu and M. Doi, J. Phys. Soc. Jpn. 53, 1031 (1984).

[12] M. Osipov and E. Terentjev, Phys. Lett. A 134, 301 (1989). 
[13] M. Osipov and E. Terentjev, Z. Naturforsch. A 44, 785 (1989).

[14] R. Larson, Macromolecules 23, 3983 (1990).

[15] P. Biscari, A. DiCarlo, and S. S. Turzi, Soft Matter 10, 8296 (2014).

[16] P. Biscari, A. DiCarlo, and S. S. Turzi, Phys. Rev. E 93, 052704 (2016).

[17] S. S. Turzi, Phys. Rev. E 94, 062705 (2016).

[18] M. Warner and E. M. Terentjev, Liquid Crystal Elastomers, International Series of Monographs on Physics (Oxford University Press, Oxford, 2003).

[19] S. S. Turzi, Eur. J. Appl. Math. 26, 93 (2015).
[20] P. de Gennes and J. Prost, Physics of Liquid Crystals, 2nd ed. (Oxford University Press, Oxford, 1995).

[21] S. S. Turzi, Phys. Rev. E 96, 052603 (2017).

[22] D.-F. Gu and A. M. Jamieson, J. Rheol. 38, 555 (1994).

[23] P. Mather, D. S. Pearson, and R. G. Larson, Liq. Cryst. 20, 539 (1996).

[24] M. E. Gurtin, E. Fried, and L. Anand, The Mechanics and Thermodynamics of Continua (Cambridge University Press, Cambridge, 2010).

[25] S. R. De Groot and P. Mazur, Non-equilibrium Thermodynamics (Dover, New York, 1984). 